\title{
A Left Pulmonary Artery Sling in an Asymptomatic Adult Patient, A Case Report and Review of Literature
}

\author{
Fang Huang, MD, Qing-Quan Lai, MD, Hong Wu, MD, Xiao-Ting Ke, MD \\ Department of Radiology, The Second Affiliated Hospital, Fujian Medical University, Quanzhou, China
}

\section{ABSTRACT}

Pulmonary artery sling (PAS) is a rare congenital vascular anomaly. Ninety percent of patients with PAS have respiratory distress and need surgical correction. Asymptomatic adult presentation of PAS is rare. We report the case of a 56-year-old female with an asymptomatic left pulmonary artery sling.

\section{INTRODUCTION}

Pulmonary artery sling (PAS) is a rare congenital cardiovascular abnormality [Yong 2013]. PSA is the abnormal origin of the left pulmonary artery from the right pulmonary artery, running between the trachea and esophagus, often combined with airway stenosis and other heart malformations. Therefore, PAS also is called the left PAS. Due to airway obstruction caused by abnormal left pulmonary artery compression, some children with PAS may have severe and life-threatening breathing difficulties in the neonatal and infant period. Some children with mild or no symptoms grow into adults, but this is rare. We report the case of an asymptomatic female with a pulmonary artery sling.

\section{CASE REPORT}

A 56-year-old female with hypertension and type 2 diabetes was referred to our radiology department for a CT pulmonary angiography (CTPA) scan, due to physical examination. The patient grew very well with no history of cardiac failure or cyanosis or repeated respiratory difficulty and pulmonary infection. There was an almost normal index in routine physical examinations, blood routine, and biochemical tests. Auscultation revealed no murmur in the chest. Transthoracic echocardiography identified an aberrant origin of the left pulmonary artery from the right pulmonary artery. CTPA revealed an anomalous left pulmonary artery originating from the right pulmonary artery with a posterior course between

Received fanuary 26, 2021; received in revised form February 11, 2021; accepted February 12, 2021.

Correspondence: Fang Huang, MD, Department of Radiology, The Second Affiliated Hospital, Fujian Medical University, Quanzhou, China (e-mail: buangfang8012@163.com). the trachea and esophagus (Figure 1). 3D reconstruction of CTPA images demonstrates the LPA from the posterosuperior portion of the RPA (Figure 2). Moreover, in addition to the fact that the left pulmonary artery originated from the right pulmonary artery, we found that the esophagus was not compressed (Figure 3). Besides, no trachea compression was found in $3 \mathrm{D}$ reconstructions from a high-resolution airway computed tomography (Figure 4). These findings might be the reason why this case survived asymptomatic to this day. This case did not have lobar dysplasia or accessory lobes, the trachea was of normal shape, and esophagus was not compressed in any way. Owing to the above condition, no surgical procedure was considered.

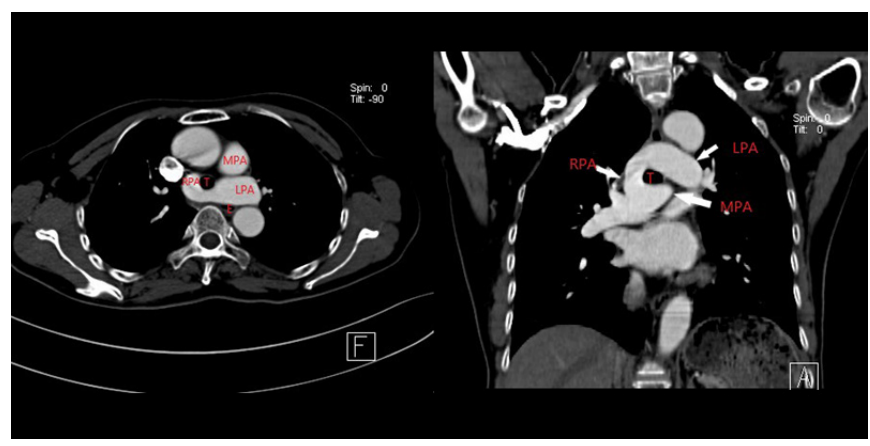

Figure 1. CT pulmonary angiography (CTPA) scan demonstrates the aberrant left pulmonary artery coursing between the trachea and esophagus. The trachea was normal in shape and without compression. (MPA, main pulmonary artery; LPA, left pulmonary artery; RPA, right pulmonary artery; $\mathrm{T}$, trachea)

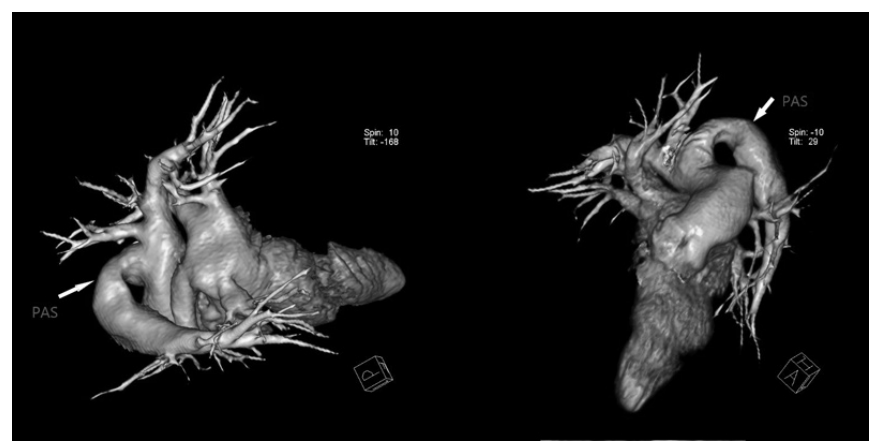

Figure 2. 3D reconstruction of CTPA images demonstrates the LPA arising from the posterosuperior portion of the RPA. (PAS, pulmonary sling) 
Literature review of adult pulmonary artery sling

\begin{tabular}{|c|c|c|c|c|c|}
\hline No. & Author & Gender & Age $(y r)$ & Cause of the finding & Diagnosis \\
\hline 1 & Nazeri 2011 & Male & 53 & Chronic cervical pain & Computed tomography \\
\hline 2 & Espinosa and Agarwal 2008 & Female & 49 & Dyspnea at 6 months & Cardiac MRI \\
\hline 3 & Lee 1996 & Female & 21 & Airway obstruction & Computed tomography \\
\hline 4 & Mammana 2020 & Male & 56 & Lung cancer & Computed tomography \\
\hline 5 & Miyazaki 2015 & Female & 33 & Dry cough and a low-grade fever & Computed tomography \\
\hline 6 & Odell 2011 & Female & 29 & Severe dyspnea and cough & Computed tomography \\
\hline 7 & LaBelle 2010 & Female & 42 & Increasing problems of dysphagia & Computed tomography \\
\hline 8 & Takahashi 2020 & Female & 44 & Esophageal squamous cell cancer & Enhanced computed tomography \\
\hline \multirow[t]{2}{*}{9} & Komoto 2013 & Male & 42 & Pharyngeal discomfort & Computed tomography \\
\hline & & Female & 36 & Bloody sputum and wheezing & Computed tomography \\
\hline
\end{tabular}

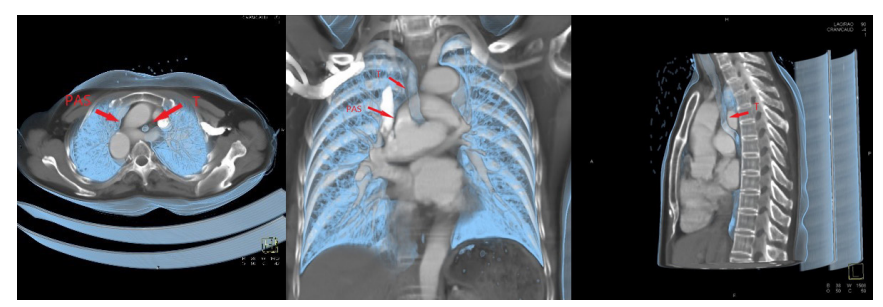

Figure 3. 3D reconstruction of CTPA images demonstrates the LPA arising from the posterosuperior portion of the RPA. The trachea was normal in shape and without compression. (PAS, pulmonary sling; T, trachea; E, esophagus)

\section{DIsCUsSION}

Pulmonary artery sling (PAS) is a rare congenital vascular anomaly [Yong 2013]. Anomalous origin of the left pulmonary artery from the right pulmonary artery is a rare congenital anomaly first described on autopsy by Glaevecke and Doehle in 1897 [Glaevecke 1897]. The term vascular sling was first used to describe the condition by Contro et al. in 1958 to distinguish it from a vascular ring [Contro 1958]. The left and right pulmonary arteries of the fetus naturally arise from either side of the lung bud and then connect with the sixth pair of aortic arcs on either side. When the left pulmonary artery cannot be connected with the left sixth arch, the left pulmonary artery is abnormal. If the sixth right arch reaches the lung bud from the caudal capillary through the midline and forms the left pulmonary plexus's connection, then the development of the left pulmonary artery lags behind the trachea's development and bronchial tree, thus forming the vascular ring [Lee 2001]. Symptoms range from absent-tosevere respiratory distress, with airway symptoms predominating in infants, whereas dysphagia may be the primary complaint of older patients [Fiore 2005]. Due to the airway obstruction caused by the left pulmonary artery's abnormal compression, some children with a pulmonary sling may have severe dyspnea or even life-threatening in the neonatal period and infancy. Therefore, early recognition and timely surgical

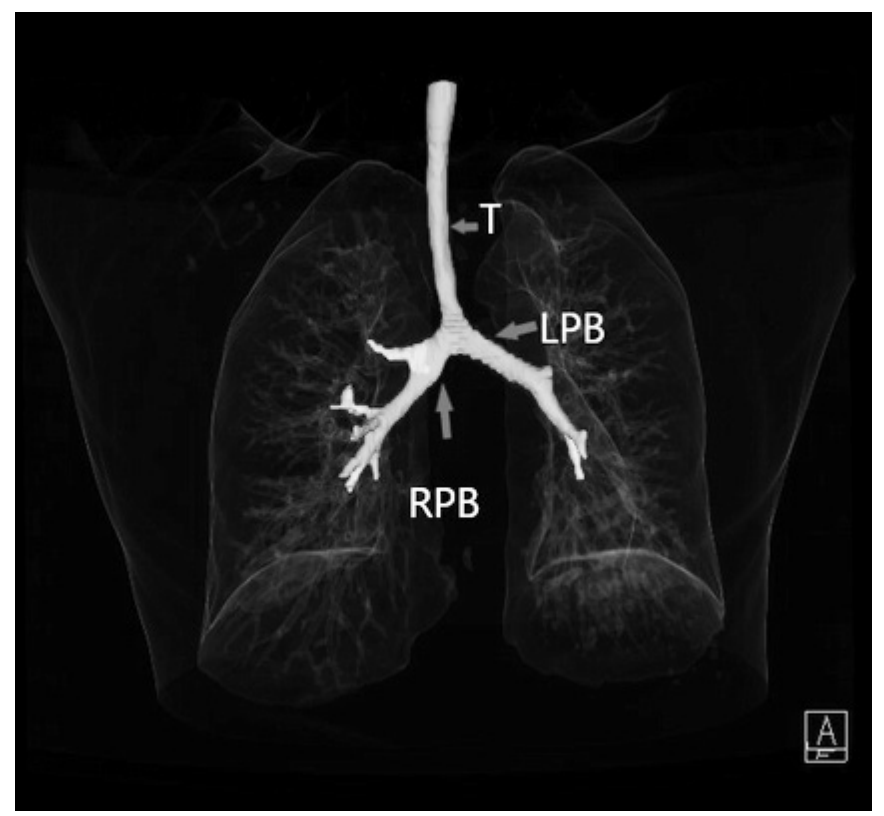

Figure 4. 3D reconstructions from a high-resolution airway computed tomography demonstrate the trachea and bronchus were normal in shape and without compression. ( $T$, trachea; LPB, left pulmonary bronchus; RPB, right pulmonary bronchus)

treatment are the keys to survival. With the improvement of the understanding of pulmonary sling and diagnostic methods, the related reports of the pulmonary sling are gradually increasing, but the indications of surgical treatment and the preoperative evaluation plan still are controversial [Hong 2015]. There may be a high mortality rate associated with PAS [Muthialu 2020]. This patient was asymptomatic because the vascular ring didn't cause compression of the trachea and esophagus. Adult presentation of PAS is rare. As far as we know, Procacci et al conducted a literature review of the adult PAS in 1993 and found a total of 17 adult patients [Procacci 1993]. After 1993, we found 10 adult patients with PAS in the literature. With this case reported, we had a total of 28 
cases in the literature, to the best of our knowledge (Table 1). Asymptomatic PAS typically is diagnosed incidentally in adolescence or adulthood. In our review of 28 adult patients with the PAS, 10 of the cases were due to the esophagus or trachea's compression. Most patients with PAS were identified due to other diseases or health examinations.

As an adjunct examination, ultrasound can be used to identify intracardiac malformation, but it is dependent on other examinations to define the left pulmonary artery and its adjacent relationship. CT and MRI play an increasingly important role in diagnosing congenital heart disease complicated with tracheal abnormality as non-invasive examinations. Although there is no ionizing radiation, MRI is not suitable for children with severe symptoms because of its long scanning time and high sedation requirements [Eichhorn 2002]. Although there is ionizing radiation in CT examination, with the emergence of MSCT, its scanning time is short, and it can be comparable to cardiovascular angiography and MRI for peripheral vascular display [Lee 2007]. Besides, with current imaging technology development, more and more children with PAS will be found at a younger age. In the literature we reviewed, CT identified the vast majority of patients. However, Espinosa and Agarwal believed that cross-sectional imaging, such as MRI and CT, was superior to echocardiography and pulmonary angiography in showing abnormalities in the left pulmonary artery, especially in the presence of pulmonary hypoplasia [Espinosa 2008].

Surgical treatment of pulmonary sling in children mainly includes left pulmonary artery reconstruction, airway stenosis correction, and intracardiac malformations treatment [Huang 2012]. With the continuous improvement of surgical techniques, the overall mortality rate has been reduced. However, PAS and tracheal stenosis treatment remain challenging, especially in the early stages after surgery. There is a higher mortality rate for children who are not treated surgically in time for symptomatic children. But Espinosa and Agarwal reported one patient, who refused surgery and survived 49 years of age [Espinosa 2008]. A female patient was diagnosed with PAS and right lung dysplasia at six months of age. The patient developed mobility limitations at an early age. With the growth of age, the symptoms gradually worsened, accompanied by dyspnea, prone to pulmonary infection, and finally, the patient died of pneumonia.

Surgery on a pulmonary artery sling in an adult is very rare. None of the 17 patients in Procacci et al.'s review received surgical treatment [Procacci 1993]. However, Mammana et al reported that an adult patient with a lung adenocarcinoma of the right upper lobe received surgical treatment [Mammana 2020]. He considered that the patient was a malignant tumor patient, the median sternal incision under off-pump circulation was adopted. A $6 \mathrm{~cm}$-long Dacron prosthesis $(22 \mathrm{~mm}$ in diameter) was used to bypass the common pulmonary trunk and the left pulmonary artery's distal portion. The operation was successful, and the patient was discharged eight days after the operation. Another adult with PAS, who underwent surgical treatment, was reported by LaBelle et al [LaBelle 2010]. The patient was a 42-year-old female, who underwent surgery for severe esophageal compression. The free left pulmonary artery was re-implanted into the main pulmonary artery under cardiopulmonary bypass through a median incision. The symptoms of esophageal compression significantly improved after the operation. To our knowledge, only two adult patients with PAS were reported to have received surgical treatment with satisfactory results. Odell et al believed that surgical correction seemed to be a safe and effective treatment for adults with severe symptoms caused by trachea and esophagus compression caused by PAS [Odell 2011].

\section{ACKNOWLEDGEMENT}

We appreciated Dr. Jiang-Shan Huang for revising our paper. We hope humans eventually defeat COVID-19.

\section{REFERENCES}

Contro S, Miller RA, White M, Potts WJ. 1958. Bronchial obstruction due to pulmonary artery abnormalities: I: vascular ring. Circulation. 17: 418-423.

Eichhorn J, Fink C, Bock M, Delorme S, Brockmeier K, Ulmer HE. 2002. Images in cardiovascular medicine. Time-resolved three-dimensional magnetic resonance angiography for assessing a pulmonary artery sling in a pediatric patient. Circulation. Oct 1;106(14):e61-2.

Espinosa L, Agarwal P. 2008. Adult presentation of right lung agenesis and left pulmonary artery sling. Acta Radiol. Feb;49(1):41-4.

Fiore AC, Brown JW, Weber TR, Turrentine MW. 2005. Surgical treatment of pulmonary artery sling and tracheal stenosis. Ann Thorac Surg. 79:38-46 discussion 38-46.

Glaevecke H, Doehle W. 1897. Ueber eine seltene angeborene anomale der pulmonalarterie. Munch Med Wochenschr. 44:950-953.

Hong X, Zhou G, Liu Y, Liu Y, Wang H, Feng Z. 2015. Management of pulmonary artery sling with tracheal stenosis: LPA re-implantation without tracheoplasty. Int J Clin Exp Med. Feb 15;8(2):2741-7.

Huang SC, Wu ET, Wang CC, Chen SJ, Chen YS, Chang CI, Chiu IS, Wang SS. 2012. Surgical management of pulmonary artery sling: trachea diameter and outcomes with or without tracheoplasty. Pediatr Pulmonol. Sep;47(9):903-8.

Komoto K, Ha-Kawa S, Tanigawa N, Kinoshita T. 2013. Two adult cases of pulmonary artery sling. Intern Med. 52(11):1271-2.

LaBelle MF, Rainer WG, Ratzer E, Miller KB. 2010. Surgical repair of pulmonary artery sling in an adult. Ann Thorac Surg. Sep;90(3):1009-11.

Lee EY. 2007. MDCT and 3D evaluation of type 2 hypoplastic pulmonary artery sling associated with right lung agenesis, hypoplastic aortic arch, and long segment tracheal stenosis. J Thorac Imaging. Nov;22(4):346-50.

Lee JC, Kim GS, Lee SJ, Yoo CG, Kim YW, Han SK, Shim YS. 1996. An adult case of pulmonary sling with complete tracheal ring. Korean J Intern Med. Jun;11(2):175-7.

Lee KH, Yoon CS, Choe KO, Kim MJ, Lee HM, Yoon HK, Kim B. 2001. Use of imaging for assessing anatomical relationships of tracheobronchial anomalies associated with left pulmonary artery sling. Pediatr Radiol. Apr;31(4):269-78.

Mammana M, Zuin A, Serra E, Bellini A, Rea F. 2020. Complex Lobectomy in a Patient With Lung Cancer and Pulmonary Artery Sling. Ann 
Thorac Surg. Jan;109(1):e55-e57.

Miyazaki T, Yamasaki N, Tsuchiya T, Matsumoto K, Hayashi H, Izumikawa K, Izumikawa K, Nagayasu T. 2015. Partial lung resection of supernumerary tracheal bronchus combined with pulmonary artery sling in an adult: report of a case. Gen Thorac Cardiovasc Surg. Mar;63(3):173-6.

Muthialu N, Martens T, Kanakis M, Bezuska L, Nakao M, Derrick G, Marek J, Khambadkone S, Kostolny M, Tsang V. 2020. Repair of pulmonary artery sling with tracheal and intracardiac defects. Asian Cardiovasc Thorac Ann. Oct;28(8):463-469.

Nazeri A, Sohawon S, Noordally SO. 2011. An asymptomatic left pulmonary artery sling and a right supernumerary tracheal bronchus. ANZ J Surg. Apr;81(4):305-6.
Odell DD, Gangadharan SP, Majid A. 2011. Pulmonary artery sling: a rare cause of tracheomalacia in the adult. J Bronchology Interv Pulmonol. Jul;18(3):278-80.

Procacci C, Residori E, Bertocco M, Di Benedetto P, Andreis IA, D'Attoma N. 1993. Left pulmonary artery sling in the adult: case report and review of the literature. Cardiovasc Intervent Radiol. Nov-Dec;16(6):388-91.

Takahashi K, Okamura A, Amada E, Otake R, Kozuki R, Toihata T, Imamura Y, Watanabe M. 2020. Esophagectomy for Esophageal Cancer in a Patient with Left Pulmonary Artery Sling. Ann Surg Oncol. May;27(5):1530.

Yong MS, d'Udekem Y, Brizard CP, Robertson T, Robertson CF, Weintraub R, Konstantinov IE. 2013. Surgical management of pulmonary artery sling in children. J Thorac Cardiovasc Surg. Apr;145(4):1033-1039. 